\title{
COMPARATIVE WEAK VALUE AMPLIFICATION AS AN APPROACH TO ESTIMATING THE VALUE OF SMALL QUANTUM MECHANICAL INTERACTIONS
}

\author{
Allen D. Parks, Scott E. Spence \\ Electromagnetic and Sensor Systems Department, 18444 Frontage Road Suite 327, Naval Surface Warfare Center Dahlgren Division, \\ Dahlgren,VA22448-5161,USA》 allen.parks@navy.mil, +1 540653 0582, scott.e.spence@navy.mil)
}

\begin{abstract}
Weak value amplification is a measurement technique where small quantum mechanical interactions are amplified and manifested macroscopically in the output of a measurement apparatus. It is shown here that the linear nature of weak value amplification provides a straightforward comparative methodology for using the value of a known small interaction to estimate the value of an unknown small interaction. The methodology is illustrated by applying it to quantify the unknown size of an optical Goos-Hänchen shift of a laser beam induced at a glass/gold interface using the known size of the shift at a glass/air interface.
\end{abstract}

Keywords: weak values, weak measurement, Goos-Hänchen shift, interaction strength, regression.

\section{Introduction}

The theoretical notion of the weak value $A_{w}$ of a quantum mechanical observable $A$ was introduced by Aharonov et al. [1-3] nearly three decades ago. This quantity is the statistical result of a standard measurement procedure performed upon a pre-selected and post-selected (PPS) ensemble of quantum systems when the interaction between the measurement apparatus and each system is sufficiently weak. Unlike the standard strong measurement of a quantum mechanical observable which significantly disturbs the measured system (i.e., collapses the wave function) and yields the mean value $\langle A\rangle$ as the measured value of $A$, a weak measurement of an observable for a PPS system does not appreciably disturb the quantum system and yields $A_{w}$ as the measured value of $A$. Unlike $\langle A\rangle-$ which is real valued and bounded by the eigenvalue spectral limits of the associated operator $\hat{A}-A_{w}$ can exhibit such eccentric properties as being complex valued and having its real part greatly exceed $\hat{A}$ 's spectral limits. While the interpretation of weak values remains somewhat controversial, several of the unusual properties predicted by the weak value theory have been experimentally verified, e.g. [4-6].

In recent years much attention has been devoted both to understanding the utility of weak values as a metrological resource, e.g. [7-13], and to using weak value amplification techniques to observe and directly measure small quantum mechanical interactions, e.g. [14-22]. This paper extends the metrological application of weak value amplification by introducing a new comparative method that exploits the linear property of weak value amplification for the purpose of quantifying an unknown value of small interactions using a known value of a similar interaction. Briefly put, measurements are used to provide a scale factor that multiplicatively transforms the known interaction's value into the value of the unknown interaction. Such scaling has the advantage of eliminating the need to convert measurement apparatus units into the appropriate units for the interaction (e.g., voltages into lengths). 
The remainder of this paper is structured as follows: the general theory of weak value amplification is summarized in the next section and used in Section 3 to develop the comparative weak value method for estimating small interaction parameters. In Section 4 the comparative method is illustrated by applying it to experimental data to estimate the unknown size of a Goos-Hänchen shift at a glass/gold interface using the known value of the shift at a glass/air interface. Concluding remarks are comprised in the final section of this paper.

\section{The theory of weak value amplification}

Weak values arise in the context of the von Neumann description of a quantum measurement at time $t_{0}$ of a time-independent observable $\hat{A}$ that describes a quantum system in an initial fixed pre-selected state $\left|\psi_{i}\right\rangle=\sum_{J} c_{j}\left|a_{j}\right\rangle$ at $t_{0}$, where the set $J$ indexes the eigenstates $\left|a_{j}\right\rangle$ of $\hat{A}$ with $\hat{A}\left|a_{j}\right\rangle=a_{j}\left|a_{j}\right\rangle$. In this description the Hamiltonian for the interaction between the measurement apparatus and the quantum system is: $\widehat{H}=\gamma(t) \hat{A} \hat{p}$.

Here $\gamma(t)=\gamma \delta\left(t-t_{0}\right)$ defines the strength of the impulsive measurement interaction at $t_{0}$ and $\hat{p}$ is the momentum operator for the pointer of the measurement apparatus which is in the initial state $|\phi\rangle$. Let $\hat{q}$ be the pointer's position operator that is conjugate to $\hat{p}$ and assume that $\langle q \mid \phi\rangle \equiv \phi(q)$ is real valued with $\langle q\rangle \equiv\langle\phi|\hat{q}| \phi\rangle=0$.

Prior to the measurement the pre-selected system and the pointer are in the tensor product state $\left|\psi_{i}\right\rangle|\phi\rangle$. Immediately following the measurement the combined system is in the state: $|\Phi\rangle=e^{-\frac{i}{\hbar} \int \hat{H} d t}\left|\psi_{i}\right\rangle|\phi\rangle=e^{-\frac{i}{\hbar} \gamma \hat{A} \hat{p}}\left|\psi_{i}\right\rangle|\phi\rangle$. If the state $\left|\psi_{f}\right\rangle,\left\langle\psi_{f} \mid \psi_{i}\right\rangle \neq 0$, is post-selected at $t_{0}$, the resulting pointer state is: $|\Psi\rangle=\left\langle\psi_{f} \mid \Phi\right\rangle=\left\langle\psi_{f}\left|e^{-\frac{i}{\hbar} \gamma \hat{A} \hat{p}}\right| \psi_{i}\right\rangle|\phi\rangle$.

A weak measurement of $\hat{A}$ occurs when the interaction strength $\gamma$ is sufficiently small so that the system is essentially undisturbed by the measurement and the pointer's position uncertainty $\Delta q$ is much larger than the separation between $\hat{A}$ 's eigenvalues. In this case, the last equation becomes $|\Psi\rangle \approx\left\langle\psi_{f}|[\hat{1}-(i / \hat{h}) \gamma \hat{A} \hat{p}]| \psi_{i}\right\rangle|\phi\rangle$ or $|\Psi\rangle \approx\left\langle\psi_{f} \mid \psi_{i}\right\rangle \hat{S}\left(\gamma A_{w}\right)|\phi\rangle$, where:

$$
A_{w} \equiv\left\langle\psi_{f}|\hat{A}| \psi_{i}\right\rangle /\left\langle\psi_{f} \mid \psi_{i}\right\rangle \equiv\left(A^{1}\right)_{w}
$$

is the complex valued weak value of observable $A$ and the operator:

$$
\hat{S}\left(\gamma A_{w}\right) \equiv e^{-\frac{i}{\hbar} \gamma A_{w} \hat{p}}
$$

is the translation operator for $|\phi\rangle$ defined by the action $\left\langle q\left|\hat{S}\left(\gamma A_{w}\right)\right| \phi\right\rangle=\phi\left(q-\gamma \operatorname{Re} A_{w}\right)$ yielding $|\langle q \mid \Psi\rangle|^{2} \approx\left|\left\langle\psi_{f} \mid \psi_{i}\right\rangle\right|^{2}\left|\phi\left(q-\gamma \operatorname{Re} A_{w}\right)\right|^{2}$ as the associated pointer distribution profile.

Thus, after the measurement the final mean pointer position is:

$$
\langle\Psi|\hat{q}| \Psi\rangle=\langle\phi|\hat{q}| \phi\rangle+\gamma \operatorname{Re} A_{w},
$$

i.e., it is translated from its initial mean position $\langle\phi|\hat{q}| \phi\rangle$ before the measurement by the amount $\gamma \operatorname{Re} A_{w}$. When $\left\langle\psi_{f} \mid \psi_{i}\right\rangle$ is small, then $\operatorname{Re} A_{w}$ can lie far outside the eigenspectral limits of $\hat{A}$, in which case $A_{w}$ is said to be amplified, and the associated pointer shift is large. Observe that when $\hat{A},\left|\psi_{i}\right\rangle$, and $\left|\psi_{f}\right\rangle$ are known, then (1) can be used to compute $A_{w}$. It is also interesting to note that under certain circumstances the imaginary part $\operatorname{Im} A_{w}$ of $A_{w}$ can be used to control the variance of the pointer state [23].

In order for measurements to qualify as weak measurements, they must be made in the weak measurement regime where the following two weakness conditions are simultaneously satisfied by the pointer's momentum uncertainty, e.g. [24]:

$$
\Delta p \ll \frac{\hbar}{\gamma}\left|A_{w}\right|^{-1}
$$




$$
\Delta p \ll \min _{(n=2,3, \cdots)} \frac{\hbar}{\gamma}\left|\frac{A_{w}}{\left(A^{n}\right)_{w}}\right|^{1 /(n-1)} .
$$

\section{A comparative weak value amplification method for estimating small interactions}

Within the weak measurement regime, if $\gamma$ is fixed and $R e A_{w}$ is considered to be an independent variable which acts as an amplification factor, then the relation given by (3) between the final pointer position and $\operatorname{Re} A_{w}$ is linear and can be represented by a straight line which has the initial mean pointer position $\langle\phi|\hat{q}| \phi\rangle$ as its "y-intercept" and $\gamma$ as its "slope". It is this linear relationship within the weak measurement regime that provides the theoretical basis for the comparative weak value estimation method developed in this section.

Suppose $M$ is an apparatus used to make a series of $m \geq 2$ weak measurements of the $m$ mean pointer positions $\langle\Psi|\hat{q}| \Psi\rangle_{i}, i=1,2, \cdots, m$, associated with $m$ independent choices of the real part $\operatorname{Re} A_{w_{i}}, i=1,2, \cdots, m$, of the weak value of an observable $A$ associated with a PPS system $\mathbb{S}$ whose interaction with $M$ 's pointer is $\gamma$. Since each such measurement is weak, then ( $i$ ) they must each simultaneously satisfy the weakness conditions given by (4a) and (4b) and (ii) the theoretical relationship between each measured mean pointer position and the associated choice made for $\operatorname{Re} A_{w}$ is governed by (3).

Let $\mathcal{S}=\left\{\left(\operatorname{Re} A_{w_{i}},\langle\Psi|\hat{q}| \Psi\rangle_{i}\right): 1 \leq i \leq m\right\}$ be the set of ordered pairs corresponding to the $m$ weak measurements performed upon $\mathbb{S}$ using the apparatus $M$. Now consider the case where $M$ is also used to make $m^{\prime} \geq 2$ weak measurements of mean pointer positions $\langle\Psi|\hat{q}| \Psi\rangle_{i}^{\prime}, i=$ $1,2, \cdots, m^{\prime}$, and associated observable weak values $\operatorname{Re} A_{w_{i}}^{\prime}, i=1,2, \cdots, m^{\prime}$, for a PPS system $\mathbb{S}^{\prime}$ whose interaction with $M^{\prime}$ 's pointer is $\gamma^{\prime}$ and let $\mathcal{S}^{\prime}=\left\{\left(\operatorname{Re} A_{w_{i}}^{\prime},\langle\Psi|\hat{q}| \Psi\rangle_{i}^{\prime}\right): 1 \leq i \leq m^{\prime}\right\}$ be the set of ordered pairs corresponding to the measurements performed upon $\mathbb{S}^{\prime}$ using $M$.

Because of the linear relationship given by (3) and provided the measurement errors are small, regression lines with large correlation coefficients can be obtained for $\mathcal{S}$ and $\mathcal{S}^{\prime}$ using standard regression software packages. Let $\langle\widetilde{\Psi|\hat{q}| \Psi\rangle}\rangle\langle\phi|\hat{q}| \phi\rangle_{0}+\gamma_{0} R e A_{w}$ and $\langle\Psi \widehat{\Psi}|\hat{q}| \Psi\rangle^{\prime}=$ $\langle\phi|\hat{q}| \phi\rangle_{0}^{\prime}+\gamma_{0}^{\prime} \operatorname{Re} A_{w}^{\prime}$ be the regression lines (denoted by “ “ " ) for $\mathcal{S}$ and $\mathcal{S}^{\prime}$, respectively, where $\langle\phi|\hat{q}| \phi\rangle_{0}$ and $\langle\phi|\hat{q}| \phi\rangle_{0}^{\prime}$ are the "y-intercepts" and $\gamma_{0}$ and $\gamma_{0}^{\prime}$ are the "slopes".

Note that since $\mathcal{S}$ and $\mathcal{S}^{\prime}$ are obtained using the same apparatus $M$, then the units of measure for each are the same. Thus, if the physical measurement unit for $M$ is $X$, then the initial and final mean pointer positions - as well as the $\gamma R e A_{w}$ terms - are quantified in $X$ units. Consequently, the slopes $\gamma$ are quantified in $X$ units per unit amplification factor. For example, if $X$ is length, then the mean pointer position and $\gamma_{0} R e A_{w}$ are in length units. As required, this is consistent with the theory developed in Section 2. In particular, consider the argument of the exponential function in (2) that defines the translation operator. There Planck's constant $\hbar$ has units: energy $\cdot$ time $=$ mass $\cdot\left(\frac{\text { length }}{\text { time }}\right)^{2} \cdot$ time $=$ mass $\cdot$ lengt $h^{2} /$ time and the linear momentum operator $\hat{p}$ has units: mass $\cdot$ length/time. Because it must be the case that the ratio $\gamma A_{w} \hat{p} / \hbar$ has no units, then $\gamma A_{w}$ must have length as its unit of measure.

If both of the slopes of the regression lines for $\mathcal{S}$ and $\mathcal{S}^{\prime}$ along with the value of one interaction strength - say $\gamma$ - are known, then the proportionality ratio $\frac{\gamma^{\prime}}{\gamma}=\frac{\gamma_{0}^{\prime}}{\gamma_{0}}$ can be used to estimate the value of the unknown interaction strength $\gamma^{\prime}$ according to the scaling:

$$
\gamma^{\prime}=\left(\frac{\gamma_{0}^{\prime}}{\gamma_{0}}\right) \gamma
$$




\section{Example: Estimating the size of an unknown Goos-Hänchen shift}

Although the law of reflection - which states that "the angle of incidence equals the angle of reflection" - is valid for plane waves (i.e., geometric optics), it is an incomplete description for transversely finite light beams which undergo total internal reflection at a dielectric interface. In this case - while the angles of incidence and reflection are equal - the center of the reflected beam is spatially translated in the plane of incidence relative to that of the reflected beam. This extremely small translation of the reflected beam is the optical Goos-Hänchen $(\mathrm{GH})$ shift $\Delta_{G H}$. If the incident beam is a superposition of linear polarizations parallel to the plane of incidence ( $p$ polarization) as well as perpendicular to it ( $s$ polarization), then the $p(s)$ polarized photons yield a reflected beam which is translated along a small distance $D_{p}\left(D_{s}\right)$ and $\Delta_{G H}=D_{p}-D_{s}$. When the angle of incidence $\alpha$ is greater than - but close to - the critical angle for total internal reflection, the GH shift of the beam is approximately given by [25]:

$$
\Delta_{G H}=\left(\frac{1}{n^{2}}-1\right)\left(\frac{n \lambda_{1}}{\pi \sqrt{\sin ^{2} \alpha-n^{2}}}\right),
$$

where $n=n_{2} / n_{1}$ and $\lambda_{1}=\lambda_{\text {vacuum }} / n_{1}$. Here $n_{1}$ is the index of refraction for the internal medium containing the incident beam, $n_{2}$ is the index of refraction for the external medium, $n_{1}>n_{2}$, and $\lambda_{\text {vacuum }}$ is the wavelength of the incident beam measured in a vacuum.

In this section the comparative weak value amplification method will be illustrated by using it to estimate the value of an unknown GH shift occurring at a glass/gold interface when the value of the corresponding GH shift at a glass/air interface is known. A schematic of the apparatus used to make the necessary series of weak measurements is shown in Fig. 1. This apparatus is similar to that used in [26] to measure GH shifts at a glass/air interface using weak value amplification.

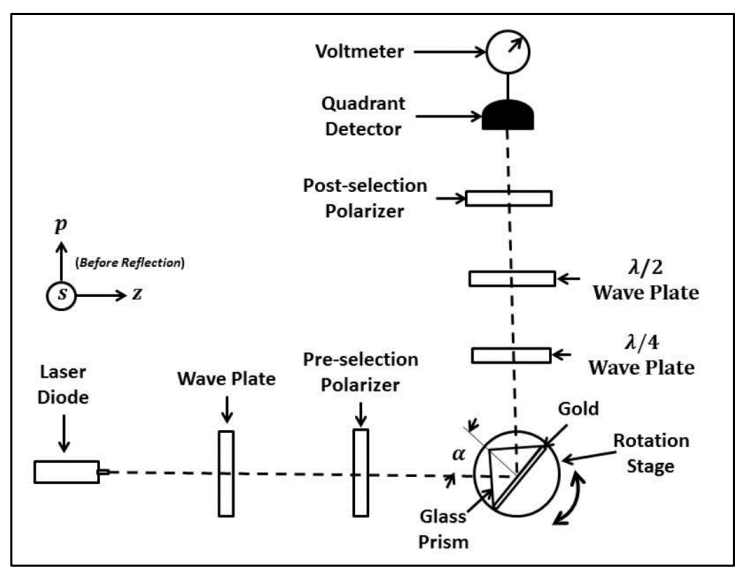

Fig. 1. A schematic of the apparatus used to make weak value measurements of $\hat{A}$.

In Fig. 1 a wave plate is used to provide an approximately equal mix of $s$ and $p$ polarized classically intense light in the Gaussian beam that is produced by a $637 \mathrm{~nm}$ wavelength laser diode with an output power of $4.8 \mathrm{~mW}$. As shown in the figure, the horizontal and vertical axes in the apparatus reference frame are labeled $p$ and $s$, respectively, with the $s$ axis pointing outward from the plane of the paper. These axes correspond to the directions of the electric field vector that define the $p$ and $s$ states of linear polarized light. A pre-selection polarizer fixes 
the initial polarization state of the light as: $\left|\psi_{i}\right\rangle=\cos \theta|s\rangle+\sin \theta|p\rangle$, with $\theta=45^{\circ}$. The beam then interacts with the interior "rear" surface of a BK7 glass prism (with and without a $50 \mathrm{~nm}$ exterior surface layer of gold ) at an incidence angle $\alpha=42^{\circ}$ and undergoes a GH shifted reflection. After traversing quarter and half wave plates which remove undesirable phase shifts and $p$ and $s$ mixing produced by the interaction, a post-selection polarizer post-selects: $\left|\psi_{f}\right\rangle=\cos \varphi|s\rangle+\sin \varphi|p\rangle$ as the polarization state of the light before it is collected by a (New Focus 2921) quadrant detector set at a $5 \mathrm{~mW}$ gain. The post-selection polarizer angle is varied in $5^{\circ}$ increments starting with $\varphi=90^{\circ}$ and ending with $\varphi=110^{\circ}$ to produce amplification of the operator to be measured. The mean of the Gaussian distribution of the beam serves as the measurement pointer of the apparatus and for each $\varphi$ value selected a (Agilent 34401A) voltmeter measures the pointer translation (which is in the plane of the paper in Fig. 1 and transverse to the beam) as the difference in the intensities of light falling upon the left and right halves (facing Fig. 1) of the detector.

The operator measured by the apparatus is the polarization operator $\hat{A}=|p\rangle\langle p|-| s\rangle\langle s|$ where $|p\rangle$ and $|s\rangle$ are orthonormal (i.e. $\langle s \mid s\rangle=\langle p \mid p\rangle=1,\langle s \mid p\rangle=\langle p \mid s\rangle=0$ ) eigenkets of $\hat{A}$ with respective eigenvalues \pm 1 . Using the orthonormal property of these eigenkets, it is determined from (1) that:

$$
A_{w}=\frac{[\cos \varphi\langle s|+\sin \varphi\langle p|][|p\rangle\langle p|-| s\rangle\langle s|][\cos \theta|s\rangle+\sin \theta|p\rangle]}{[\cos \varphi\langle s|+\sin \varphi\langle p|][\cos \theta|s\rangle+\sin \theta|p\rangle]}=\frac{\sin \varphi \sin \theta-\cos \varphi \cos \theta}{\cos \varphi \cos \theta+\sin \varphi \sin \theta} .
$$

It is convenient to write $A_{w}$ as a function of an offset angle $\omega$ referenced to the fixed preselection angle $\theta=45^{\circ}$. Using $\omega=\varphi-\theta=\varphi-45^{\circ}$ in the last equation yields $A_{w}=\tan \omega$ as the amplification factor. Also, from [26] it is found that the interaction strength is $\gamma=$ $(1 / 2) \Delta_{G H}$ in which case the pointer translation is $\gamma R e A_{w}=\frac{1}{2} \Delta_{G H} \tan \omega$.

Thus, for each offset angle $\omega=45^{\circ}, 50^{\circ}, 55^{\circ}, 60^{\circ}$ and $65^{\circ}$, the interaction strength is amplified by the weak value $\tan \omega=1.000,1.192,1.428,1.732$ and 2.144 , respectively.

It is necessary to obtain the weakness condition that must be satisfied in order that the measurements of $A$ are sufficiently weak so that (3) applies. Observe from (4b) that when $n$ is odd, $\left(A^{n}\right)_{w}=A_{w}$ and when $n$ is even, $\left(A^{n}\right)_{w}=1$. Applying these results and the Heisenberg uncertainty relation $\Delta q \Delta p \geq \hbar / 2$ to $(4 a)$ and ( $4 b)$ gives $\Delta q \gg \frac{1}{4} \Delta_{G H} \tan \omega$ and

$$
\Delta q \gg\left\{\begin{array}{c}
\frac{1}{4} \Delta_{G H}, n \text { odd } \\
\frac{1}{4} \Delta_{G H}\left[\min _{n=2,4,6, \cdots}(\tan \omega)^{1 /(n-1)}\right]^{-1}, n \text { even }
\end{array},\right.
$$

respectively, where $\Delta q$ is the uncertainty in the pointer's (horizontal) position. Since $1.000 \leq$ $\tan \omega \leq 2.144$, then it is clear that the single inequality $\Delta_{G H} \ll 4 \Delta q \cot \omega$ simultaneously satisfies (4a) and (4b) and defines the weak measurement regime. Using in this inequality the reasonable assumption that the pointer's position uncertainty $\Delta q$ is to a good approximation the $\sim 1 \mathrm{~mm}=10^{6} \mathrm{~nm}$ width of the laser beam yields the following weakness condition that must be satisfied by the Goos-Hänchen shift for measurements made in the offset angle range $45^{\circ} \leq$ $\omega \leq 65^{\circ}$ :

$$
\Delta_{G H} \ll 4 \times 10^{6} \cot \omega n m .
$$

Before performing the desired series of measurements for both the glass/air and the glass/gold cases, the zero reference voltage was determined by centering the laser beam intensity distribution on the quadrant detector using $\theta=\varphi=45^{\circ}$. The results for each series of measurements relative to the associated zero reference voltages are presented in Fig. 2. 


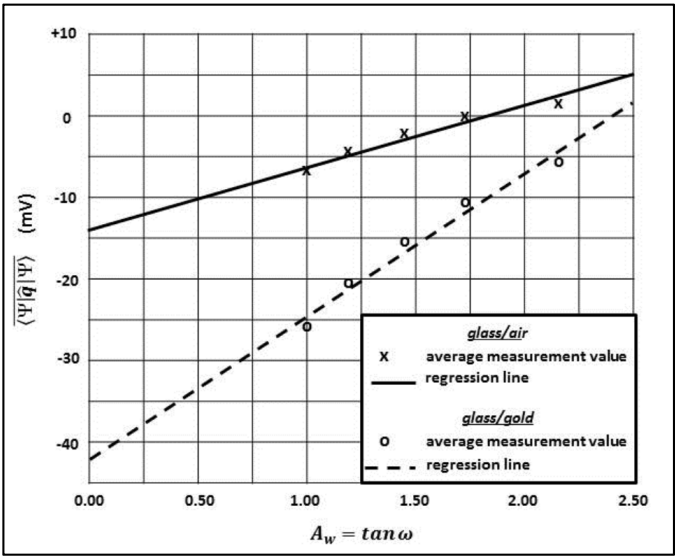

Fig. 2. Average measurement values $\overline{\langle\Psi|\hat{q}| \Psi\rangle}$ vs. $A_{w}$ and regression lines.

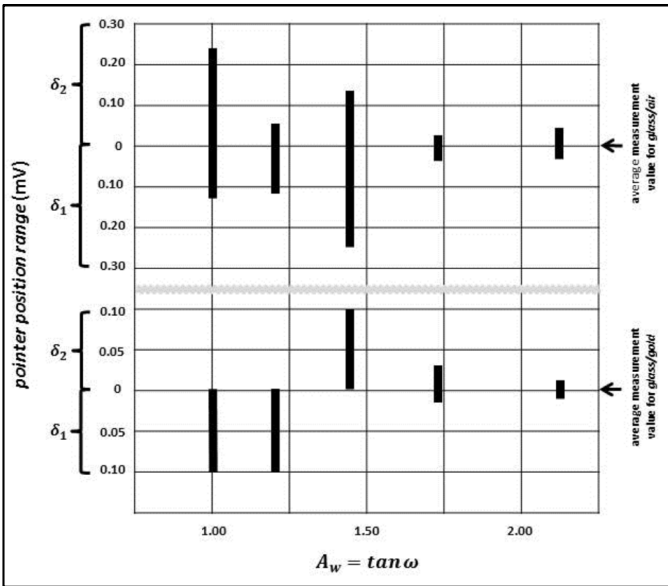

Fig. 3. The ranges for the dispersion of pointer position measurements around average pointer positions $\overline{\langle\Psi|\hat{q}| \Psi\rangle}$ vs. $A_{w}$.

Each measurement result is an average $\overline{\langle\Psi|\hat{q}| \Psi\rangle}$ of $\sim 185$ distinct pointer positions, where each pointer position corresponds to a single voltage reading provided by the paired voltmeter/quadrant detector used in the apparatus. The $\sim 185$ pointer positions comprising all averages were collected during a 60 second time interval (this interval was selected based upon previous experience using this voltmeter/quadrant detector pair since it was found that increasing the interval had a negligible effect on measurement results). The average measurement value $\overline{\langle\Psi|\hat{q}| \Psi\rangle}$, along with the minimum and maximum pointer position values used in the average, were automatically generated by the voltmeter. These minimum and maximum values provide a measure of the dispersion of the pointer position values used to compute the associated average. They were converted into range brackets $\left[\delta_{1}, \delta_{2}\right]$ for each average measurement value and indicate that all measurements used to compute a measurement average $\overline{\langle\Psi|\hat{q}| \Psi\rangle}$ had values in the range $\left[\overline{\langle\Psi|\hat{q}| \Psi\rangle}-\delta_{1}, \overline{\langle\Psi|\hat{q}| \Psi\rangle}+\delta_{2}\right] \mathrm{mV}$. Since this range for each measurement average is smaller than the symbols used to represent the data points 
in Fig. 2, they are reported separately in Fig. 3 as vertical bars representing the dispersion of pointer position measurements around the average measured value $\overline{\langle\Psi|\hat{q}| \Psi\rangle}$.

The regression line $\langle\widetilde{\Psi|\hat{q}| \Psi}\rangle_{\text {glass } / \text { air }}=-13.4+7.30$ tan $\omega \mathrm{mV}$ with a correlation coefficient of 0.968 , a slope uncertainty of $\sigma_{0}=0.89 \mathrm{mV}$, and a y-intercept uncertainty of $\sigma_{1}=1.4 \mathrm{mV}$ was obtained for the five point glass/air measurement data set and the regression line $\langle\widehat{\Psi|\hat{q}| \Psi}\rangle_{\text {glass } / \text { gold }}=-42.2+17.6 \tan \omega \mathrm{mV}$ with a correlation coefficient of 0.982 , a slope uncertainty of $\sigma_{0}^{\prime}=1.6 \mathrm{mV}$, and a y-intercept uncertainty of $\sigma_{1}^{\prime}=2.4 \mathrm{mV}$ was obtained for the five point glass/gold data set. These regression lines are presented along with the measurement data in Fig. 2. Note that the y-intercepts $-13.4( \pm 1.4) \mathrm{mV}$ and $-42.2( \pm 2.4) \mathrm{mV}$ of the two regression lines correspond to the theoretical average initial pointer positions for the glass/air and glass/gold measurement sets. However, recall from above that the experimental zero reference voltage for the pointer position was obtained by centering the intensity of the laser beam on the quadrant detector.

Thus, the slopes expressed in apparatus measurement units are $\gamma_{\text {glass/air }}=\gamma_{0} \pm \sigma_{0}=7.30 \pm$ $0.89 \mathrm{mV}$ and $\gamma_{\text {glass } / \text { gold }}=\gamma_{0}^{\prime} \pm \sigma_{0}^{\prime}=17.6 \pm 1.6 \mathrm{mV}$.

Since $\alpha=42^{\circ}$ is near but greater than the critical angle for the glass/air interface (i.e., $\sim 41.8^{\circ}$ ), (6) can be used to estimate $\Delta_{G H, \text { glass } / \text { air }}=1786 \mathrm{~nm}$. (It is important to note that: (6) should not be used to evaluate $\Delta_{G H, g l a s s / g o l d}$ because the associated critical angle is not near $\alpha$; and $\Delta_{G H, \text { glass/gold }}$ is likely enhanced by a surface plasmon resonance effect occurring at $\alpha \approx 44^{\circ}$, e.g. [27]). Application of (5) provides the desired estimated result (the $1 / 2$ factors in $\gamma$ and $\gamma^{\prime}$ cancel one another): $\Delta_{G H, \text { glass } / \text { gold }}=\left(\frac{17.6 \mathrm{mV}}{7.30 \mathrm{mV}}\right)(1786 \mathrm{~nm})=4304 \mathrm{~nm}$.

It remains to verify that the weakness condition (7) is satisfied for both series of measurements. For the range of offset angles $\omega$ used in both series of measurements, the smallest value for the right hand side of (7) occurs at $\omega=65^{\circ}$ so that $\Delta_{G H} \ll 1869 \times 10^{3} \mathrm{~nm}$. By inspection it is readily seen that both $\Delta_{G H, \text { glass / air }}$ and $\Delta_{G H, \text { glass/gold }}$ satisfy this inequality. Consequently, both series of measurements qualify as weak measurements.

It is important to point out that although the weakness condition (7) is not violated for $\omega=$ $65^{\circ}$, amplification factors greater than 2.14 were not used here. This is because the average measurement values begin to sharply depart from the linear behavior associated with weak value measurements when $\omega=65^{\circ}$. This is primarily a consequence of the fact that the pointer distribution profile - which is effectively a broad Gaussian peaked at $\gamma \operatorname{Re} A_{w}=\frac{1}{2} \Delta_{G H} \tan \omega$ when $\omega \leq 65^{\circ}$ - starts to become bimodal when $\omega>65^{\circ}$ with a second peak appearing to the left of the original peak. As $\omega$ approaches $90^{\circ}$ the light becomes more equally distributed around each peak and the detector voltage difference approaches zero. This behavior can be seen in Fig. 2 in [5] and Figs. 2-4 in [28] and is discussed in [29] from a statistical mixture perspective for projection operators as the measurement interaction strength increases.

\section{Concluding remarks}

This paper introduces a new weak value based methodology that can be used to estimate the unknown value of a small quantum mechanical interaction from an interaction with a known value. The method is straightforward, eliminates the need to convert measurement units into interaction units (as is generally the case for direct measurements of the interaction), and capitalizes upon the linear translation of the pointer of the measurement apparatus as the weak value of the observable is amplified. It requires: $(i)$ a series of weak value measurements of an observable for the case where the interaction is known and for the case where it is unknown; (ii) knowledge of the operator that represents the observable to be measured, as well as the associated PPS states, in order to evaluate the amplification factor and weakness condition 
for each measurement; (iii) that each such measurement qualifies as a weak measurement by satisfying an associated weakness condition; and (iv) fitting the slopes of the regression lines to each series of measurements. The estimate of the value of the unknown interaction is obtained by scaling the known interaction value by the ratio of these slopes. It can also be used to establish ratios of interaction values when both their values are unknown or unobtainable (in certain applications it may be the case that ratios are adequate). The method is illustrated by using it to estimate the optical Goos-Hänchen shift at a glass/gold interface using the known shift at a glass/air interface.

In closing, it is interesting to entertain the possibility that a methodology similar to that discussed in this paper might be developed for non-linear theories of order $\gamma^{n}, n>1$. Although theories for all orders have been studied for PPS systems, e.g. [29-31], due to the apparent additional complexities associated with such methodologies, the advantage of applying nonlinear theories to comparatively determine small interactions is not obvious. It is clear that additional research is needed to evaluate the metrological utility of such an approach.

\section{Acknowledgment}

This work was supported by a grant from the Naval Surface Warfare Center Dahlgren Division's In-house Laboratory Independent Research Program.

\section{References}

[1] Aharonov, Y., Albert, D., Casher, A., Vaidman, L. (1986). New Techniques and Ideas in Quantum Measurement Theory. New York: New York Academy of Science.

[2] Aharonov, Y., Albert, D., Vaidman, L. (1988). How the result of a measurement of a component of the spin of a spin-1/2 particle can turn out to be 100. Phys. Rev. Lett., 60, 1351-1354.

[3] Aharonov, Y., Vaidman, L. (1990). Properties of a quantum system during the time interval between two measurements. Phys. Rev. A, 42, 11-20.

[4] Ritchie, N., Storey, J., Hulet, R. (1991). Realization of a measurement of a "weak value". Phys. Rev. Lett., 66, 1107-1110.

[5] Parks, A., Cullin, D., Stoudt, D. (1998). Observation and measurement of an optical Aharonov-AlbertVaidman effect. Proc. R. Soc. Lond. A, 454, 2997-3008.

[6] Wang, Q., Sun, F., Zhang, Y., Jian, L., Huang, Y. Guo, G. (2006). Experimental demonstration of a method to realize weak measurement of the arrival time of a single photon. Phys. Rev. A, 73, 023814.

[7] Hofmann, H. (2011). Uncertainty limits for quantum metrology obtained from the statistics of weak measurements. Phys. Rev. A, 83, 022106.

[8] Hayat, A., Feizpour, A., Steinberg, A. (2011). Enhancing metrological sensitivity by weak measurements. SPIE Newsroom, DOI:10.1117/2.1201112.004014.

[9] Ferrie, C., Combes, J. (2014). Weak Value Amplification is Suboptimal for Estimation and Detection. Phys. Rev. Lett., 112, 040406.

[10] Jordan, A., Martínez-Rincón, J., Howell, J. (2014). Technical Advantages for Weak-Value Amplification: When Less Is More. Phys. Rev. X, 4, 011031.

[11] Knee, G., Gauger, E. (2014). When Amplification with Weak Values Fails to Suppress Technical Noise Phys. Rev. X, 4, 011032.

[12] Alves, G., Escher, B., Filho, R., Zagury, N., Davidovich, L. (2014). Weak-value amplification as an optimal metrological protocol. arXiv: 1410.7415v1 [quant-ph]

[13] Zhang, L., Datta, A., Walmsley, I. (2015). Precision Metrology Using Weak Measurements. Phys. Rev. Lett., $114,210801$. 
[14] Hosten, O., Kwiat, P. (2008). Observation of the Spin Hall Effect of Light via Weak Measurements. Science, $319,787$.

[15] Hoffman, H. (2010). On the estimation of interaction parameters in weak measurements. arXiv: 1012.007v1 [quant-ph]

[16] Starling, D., Dixon, P., Jordan, A., Howell, J. Precision frequency measurements with interferometric weak values. Phys. Rev. A, 82, 063822 .

[17] Howell, J., Starling, D., Dixon, P., Vudyasetu, P., Jordan, A. (2010). Interferometric weak value deflections: Quantum and classical treatments. Phys. Rev. A, 81, 033813.

[18] Pfeifer, M., Fischer, P. (2011). Weak value amplified optical activity measurements. OPTICS EXPRESS, 19, 16508.

[19] Parks, A., Spence, S. (2012). Weak value amplification of an optical Faraday differential refraction effect. APPLIED OPTICS, 51, 3364-3369.

[20] Gorodetski, Y., Bliokh, K., Stein, B., Genet, C., Shitrit, N., Kleiner, V., Hasman, E., Ebbesen, T. (2012). Weak measurements of Light Chirality with a Plasmonic Slit. Phys. Rev. Lett., 109, 013901.

[21] Hoffman, H., Goggin, M., Almeida, M., Barbieri, M. (2012). Estimation of a quantum interaction parameter using weak measurements: Theory and experiment. Phys. Rev. A, 86, 040102(R)

[22] Viza, G., Martínez-Rincón, J., Alves, G., Jordan, A., Howell, J. (2015). Experimentally quantifying the advantages of weak-value-based metrology. Phys. Rev. A, 92, 032127.

[23] Parks, A., Gray, J. (2011). Variance control in weak value measurement pointers. Phys. Rev. A, 84, 012116

[24] Spence, S., Parks, A. (2012). Experimental evidence for a dynamical non-locality induced effect in quantum interference using weak values. Found. Phys., 42, 803-815.

[25] Renard, R. (1964). Total reflection: A new evaluation of the Goos-Hänchen shift. J. Opt. Soc. Am., 54, 1190-1197.

[26] Jayaswal, G., Mistura, G., Merano, M. (2013). Weak measurements of the Goos-Hänchen shift. Opt. Lett., 38, 1232-1234.

[27] Yin, X., Hesselink, L., Liu, Z., Fang, N., Zhang, X. (2004). Large positive and negative lateral optical beam displacements due to surface plasmon resonance. Appl. Phys. Lett., 85, 372-374.

[28] Duck, I., Stevenson, P., Sudarshan, E. (1989). The sense in which a "weak measurement" of a spin-1/2 particle's spin component yields a value 100. Phys. Rev. D, 40, 2112-2117.

[29] Parks, A., Spence, S., Gray, J. (2014). Exact pointer theories for von Neumann projector measurements of pre- and postselected and preselected-only quantum systems: statistical mixtures and weak value persistence. Proc. R. Soc. A, 470, 20130651.

[30] Wu, S., Li, Y. (2011). Weak measurements beyond the Aharonov-Albert-Vaidman formalism. Phys. Rev. A, 83, 052106

[31] Nakamura, K., Nishizawa, A., Fujimoto, M. (2012). Evaluation of weak measurements to all orders. Phys. Rev. $A, 85,012113$. 